\title{
A Continuum model for skeletal muscle contraction at homogeneous finite deformations
}

\author{
Babak Sharifimajd and Jonas Stålhand
}

\section{Linköping University Post Print}

\section{Tweet}

N.B.: When citing this work, cite the original article.

The original publication is available at www.springerlink.com:

Babak Sharifimajd and Jonas Stålhand, A Continuum model for skeletal muscle contraction at homogeneous finite deformations, 2013, Biomechanics and Modeling in Mechanobiology, (12), 5, 965-973.

http://dx.doi.org/10.1007/s10237-012-0456-x

Copyright: Springer Verlag (Germany)

http://www.springerlink.com/?MUD=MP

Postprint available at: Linköping University Electronic Press

http://urn.kb.se/resolve?urn=urn:nbn:se:liu:diva-85805 


\title{
A continuum model for skeletal muscle contraction at homogeneous finite deformations
}

\author{
Babak Sharifimajd · Jonas Stålhand
}

Received: date / Accepted: date

\begin{abstract}
In this paper we present a homogeneous continuum mechanical model for the active behavior of the skeletal muscle under finite strains. The model differs from other skeletal muscle models in the way which the contractile force is introduced. Generally, the contractile force is postulated to be the isometric force multiplied by a set of experimentally motivated functions which account for the muscles active properties. Although both flexible and simple, this approach does not automatically guarantee a thermodynamically consistent behavior but this must be checked in each case. The model proposed herein is derived from fundamental principles in mechanics using a previously presented framework (Stålhand et al., Prog Biophys Molec Biol, 96, 2008) and implicitly guarantees a dissipative and thermodynamically consistent behavior. To show the performance of the model, it is specialized to a quick-release experiment for rabbit tabialis anterior muscle. The results show that the model is able to capture important characteristics like the bell-shaped force-length curve and hyperbolic force-velocity relation.
\end{abstract}

Keywords Skeletal muscle · Model · Dissipation inequality · Strain-energy function · Continuum mechanics.

\section{Introduction}

The skeletal muscle is a central component in the musculoskeletal system and provides mammals with an ability to carry out essential functions like breathing, chewing, handling objects, standing, walking etc. It is therefore no surprise that the active properties of skeletal muscles have attracted attention and been thoroughly studied. Much of what is known today about the active properties

Babak Sharifimajd · Jonas Stålhand

Mechanics, Department of Management and Engineering, The Institute of Technology, Linköping University, SE-581 83, Linköping, Sweden

E-mail: babak.sharifimajd@liu.se 
was discovered during the first half of the 20th century thanks to scientists like W.O. Fenn, A.M. Gordon, A.V. Hill, and A.F. Huxley (McMahon 1984).

Among the wealth of information on skeletal muscle in the literature, a few principle characteristics are commonly included in muscle modeling, e.g., the bell-shaped length dependence of the developed force and the hyperbolic relation between shortening velocity and afterload. The hyperbolic relation is often referred to as the Hill equation after its discoverer and is given by the equation

$$
V=b \frac{t_{0}-t}{t+a}
$$

where $V$ is the shortening velocity, $t$ the (isotonic) afterload, $t_{0}$ the isometric stress, and $a, b>0$ are constants. These characteristics are linked to the mechanics of the muscle and any kinetic study of, e.g., mammal motion must also include a mechanical model for the muscle. One of the first to suggest such a model was A.V. Hill (Hill, 1938). Based on amazingly accurate measurements of the temperature during muscle contraction, he concluded that the muscle can be represented by a spring in series with a contractile element governed by Eq. (1). Despite its simplicity, this model is able to explain most of the active properties and is still used in some studies (Fung 1993 and Martins et al. 1998), or has served as a base for more refined models (Zajac 1989, Meijer et al. 1998, and Ettema and Meijer 2000).

A common strategy to model the active muscle is to define the developed force constitutively in such a way that it captures the characteristics above. This is often done by taking the developed force $F$ to be the product of the isometric force $F_{0}$ and some experimentally motivated functions. For example, in a quick-release experiments, one may choose the normalized functions to be $f(L)$ and $g(V)$ (both in the range $[0,1]$ ) describing the dependence on length $L$ and velocity $V$, respectively, see, e.g., Buchanan et al. (2004), Johansson et al. (2000) and Liang et al. (2006). These functions are, more or less, arbitrary which makes the method both flexible and simple, but also introduces some weaknesses. To guarantee a physically reasonable behavior, the model must fulfill requirements like dissipation and objectivity. This is not implicitly guaranteed when the force is postulated as above and may lead to restrictions in the model, see Ambrosi and Pezzuto (2012) and Stålhand et al. (2008).

The objective of this study is to derive a skeletal muscle model which satisfies the requirements above. This is done by applying fundamental principles in mechanics using a previously presented framework (see Stålhand et al. 2008, and Stålhand et al. 2011). This framework implicitly guarantees the model to be dissipative and thermodynamically consistent. The performance of the derived model is validated by tailoring it to quick-release experiments for a rabbit tibialis anterior muscle. The model is confined to homogeneous strain fields and fully tetanized conditions to limit the complexity. Further, aspects such as pennation angle and residual force enhancement (McMahon 1984; Edman et al. 1982) are also excluded herein. 


\section{Constitutive modeling}

\subsection{Kinematics}

Consider the three-component mechanical model for skeletal muscle contraction given in Fig. 1. The force generating unit consists of a friction-clutch in series with a spring where the friction-clutch represents the force generated by the power strokes and the spring represents the cross-bridge elasticity. The serial spring may also include other elastic structures in a serial arrangement with the cross-bridges, e.g., the filaments themselves or titin, but all these effects are lumped into the same spring for simplicity. Parallel to the force generating unit is an elastic spring representing passive structures such as connective tissue.

Let the length of the reference state be $l_{0}$ and assume a homogeneous deformation comprising two fictitious steps: first, an active displacement $u_{a}$ which slides (translates) the filaments relative to each other without deforming the cross-bridges and, second, a displacement $u_{e}$ which stretches the crossbridges without sliding the filaments. The deformed length is then given by $l=l_{0}+u_{a}+u_{e}$ and the total stretch can be written

$$
\lambda=\frac{l}{l_{0}}=1+\varepsilon_{a}+\varepsilon_{e}
$$

where $\varepsilon_{a}=u_{a} / l_{0}$ and $\varepsilon_{e}=u_{e} / l_{0}$. The time derivative of Eq. (2) reads

$$
\dot{\lambda}=\dot{\varepsilon}_{a}+\dot{\varepsilon}_{e}
$$

where a superscribed dot denotes time derivative.

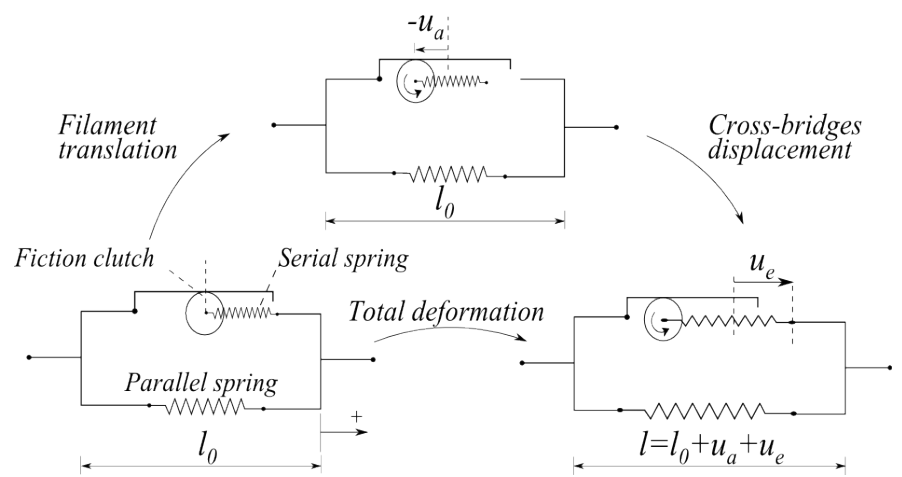

Fig. 1 The kinematics of skeletal muscle contraction. $l_{0}$ is the reference length, $l$ is the deformed length, $u_{a}$ is the translation (sliding) of filaments and $u_{e}$ is the displacement of cross-bridges.

In addition to the deformation rates in Eq. (3), we also define the relative deformation rate between the clutch and sliding filaments

$$
w=\dot{\varepsilon}_{a}+v,
$$


where $v$ can be thought as the normalized velocity for the clutch given by

$$
v=\frac{r}{l_{0}} \dot{\theta}
$$

where $r$ is the clutch radius and $\dot{\theta}$ its angular velocity.

\subsection{Balance laws}

To derive balance laws, the principle of virtual power (Germain 1973) is used. The state variables for this problem are taken to be $\lambda, \varepsilon_{a}$ and $\theta$, and the virtual velocities of the state variables are denoted $\delta \lambda, \delta \varepsilon_{a}$ and $\delta \theta$, respectively. Using the virtual velocities, the internal virtual power for the mechanical model can be written

$$
\hat{P}_{i n t}=T \delta \lambda+T_{e} \delta \varepsilon_{e}+T_{a}\left(r \delta \theta / l_{0}+\delta \varepsilon_{a}\right),
$$

where $T, T_{e}$, and $T_{a}$ are the internal thermodynamic forces power conjugate to $\delta \lambda, \delta \varepsilon_{e}$, and $r \delta \theta / l_{0}+\delta \varepsilon_{a}$, respectively. The first and second terms in Eq. (6) are the powers associated with passive structures and cross-bridges, respectively. Note that $\delta \varepsilon_{e}$ is not an independent state variable and can been replaced by $\delta \lambda-\delta \varepsilon_{a}$ using Eq. (3). The last term relates to the power expended on the friction-clutch. Next, the external power of the mechanical model can be written

$$
\hat{P}_{e x t}=t \delta \lambda+t_{a} r \delta \theta / l_{0},
$$

where $t$ and $t_{a}$ are the external thermodynamic forces power conjugate to $\delta \lambda$ and $r \delta \theta / l_{0}$, respectively. The first term in Eq. (7) is the power associated with the external deformation of the muscle while the second term can be thought as the external power applied to drive the friction clutch. The principle of virtual power requires

$$
\hat{P}_{\text {int }}=\hat{P}_{\text {ext }},
$$

for all admissible virtual velocity fields. By substituting Eqs. (6) and (7) into Eq. (8) and rearranging the terms, we get

$$
\left(T+T_{e}-t\right) \delta \lambda+\left(T_{a}-T_{e}\right) \delta \varepsilon_{a}+\left(T_{a}-t_{a}\right) r \delta \theta / l_{0}=0 .
$$

Since the virtual velocity fields are arbitrary, the following balance laws must hold:

$$
\begin{gathered}
t=T+T_{e}, \\
T_{a}=T_{e}, \\
t_{a}=T_{a} .
\end{gathered}
$$




\subsection{Constitutive relations}

To derive constitutive equations, we apply the dissipation inequality which states

$$
\dot{\Psi} \leq P_{\text {int }}
$$

for all admissible velocity fields. In Eq. (13) $\Psi$ is the free energy and $P_{\text {int }}$ is the internal power obtained by replacing the virtual velocities in Eq. (6) for their true counterparts. Following Stålhand et al. (2008), the free energy of the model is assumed to be additively decomposed in the following way:

$$
\Psi=\Psi^{p}(\lambda)+N\left(\varepsilon_{a}\right) \Psi^{e}\left(\varepsilon_{e}\right),
$$

where $\Psi^{p}$ is the elastic energy in the passive structures, $\Psi^{e}$ is elastic energy in the cross-bridges, and $N\left(\varepsilon_{a}\right) \in[0,1]$ is a function describing the effective fraction of overlap between actin and myosin filaments.

Substituting $P_{\text {int }}$ and $\Psi$ in the dissipation inequality (13) and using Eq. (4) gives

$$
\frac{\partial \Psi^{p}}{\partial \lambda} \dot{\lambda}+\frac{\partial N}{\partial \varepsilon_{a}} \Psi^{e} \dot{\varepsilon}_{a}+N \frac{\partial \Psi^{e}}{\partial \varepsilon_{e}}\left(\dot{\lambda}-\dot{\varepsilon}_{a}\right) \leq T \dot{\lambda}+T_{e}\left(\dot{\lambda}-\dot{\varepsilon}_{a}\right)+T_{a} w
$$

and after rearranging of the terms

$$
\left(T+T_{e}-\frac{\partial \Psi^{p}}{\partial \lambda}-N \frac{\partial \Psi^{e}}{\partial \varepsilon_{e}}\right) \dot{\lambda}+\left(-T_{e}-\frac{\partial N}{\partial \varepsilon_{a}} \Psi^{e}+N \frac{\partial \Psi^{e}}{\partial \varepsilon_{e}}\right) \dot{\varepsilon}_{a}+T_{a} w \geq 0 .
$$

The first term in Eq. (16) is assumed to be associated with elastic processes in the springs in Fig. 1 and is taken to be

$$
T+T_{e}-\frac{\partial \Psi^{p}}{\partial \lambda}-N \frac{\partial \Psi^{e}}{\partial \varepsilon_{e}}=0
$$

The second term is related to the filament sliding. This makes it natural to assume a dissipative process and take

$$
-T_{a}-\Psi^{e} \frac{\partial N}{\partial \varepsilon_{a}}+N \frac{\partial \Psi^{e}}{\partial \varepsilon_{e}}=\eta \dot{\varepsilon}_{a}
$$

where $\eta>0$ and Eq. (11) has been substituted. The third term is the stress applied to the system by the friction clutch and it is assumed to be linearly dependent on the relative deformation rate in Eq. (4) and on the filament overlap, i.e.,

$$
T_{a}= \begin{cases}N \kappa\left(v+\dot{\varepsilon}_{a}\right) & \text { if } \dot{\varepsilon}_{a} \geq-v \\ 0 & \text { if } \dot{\varepsilon}_{a}<-v\end{cases}
$$

where $\kappa>0$ is a constant and Eq. (4) has been substituted. In Eq. (19), it is further assumed that the sliding velocity $\dot{\varepsilon}_{a}$ cannot exceed the normalized velocity of the clutch in contractions and, as a consequence, $T_{a}$ is taken to be zero whenever $\dot{\varepsilon}_{a}<-v$. Note that these choices make the contraction dissipative. This is easily verified by back-substituting Eqs. (17) to (19) into 
(16). The resulting inequality is greater than zero for all non-zero $\dot{\varepsilon}_{a}$ and $w$ which guarantees a dissipative contraction.

Finally, by substituting Eq. (10) into Eq. (17), we obtain

$$
t=\frac{\partial \Psi^{p}}{\partial \lambda}+N \frac{\partial \Psi^{e}}{\partial \varepsilon_{e}} .
$$

Equations (18) to (20) constitute the general model for skeletal muscle contraction. In order to specialize the model, the strain-energy functions, $N(\varepsilon)$, and $\eta$ must be specified.

\subsection{Specific constitutive model}

It is generally accepted that the uniaxial stress response of passive skeletal muscle is an exponential function with respect to the total stretch, see e.g., (Ehret et al. 2011; Ehret and Itskov 2007). In this regard, we take $\Psi^{p}$ to be

$$
\Psi^{p}=\frac{C_{1}}{2 C_{2}}\left(\exp \left(C_{2}\left(\lambda^{2}-1\right)^{2}\right)-1\right),
$$

where $C_{1}>0$ and $C_{2}>0$ are material constants. The strain energy of the cross-bridges is assumed to be

$$
\Psi^{e}=\frac{1}{2} K_{e} \varepsilon_{e}^{2},
$$

where $K_{e}>0$ is the stiffness of the serial spring.

The developed force in a skeletal muscle is a nonlinear function of the muscle length with the maximal force close to its in situ length. The length at which the muscle develops its maximal force is usually referred to as the 'optimal muscle length' (McMahon 1984) and it has been shown that the optimal length is related to the maximal degree of overlap between myosin and actin filaments in individual sarcomeres (Gordon et al. 1966). The overlap function $N\left(\varepsilon_{a}\right)$ herein is assumed to be dependent on the filament sliding according to

$$
N=\exp \left(-\left(\varepsilon_{a}^{o p t}-\varepsilon_{a}\right)^{2} /\left(2 \gamma^{2}\right)\right),
$$

where $\varepsilon_{a}^{o p t}$ is the value for $\varepsilon_{a}$ at maximum overlap and $\gamma>0$ is a constant.

What remains to be specified is $\eta$ in Eq. (18). Although it is possible to treat $\eta$ as an unknown constant and identify it directly from time-dependent experiments, we will not follow this path here. Instead, we will choose a particular functional form for $\eta$ and specialize Eq. (18) in the intent to recover a form of the Hill equation in (1). To that end, we start by substituting Eq. $(19)^{1}$ into (18) and eliminating $\partial \Psi^{e} / \partial \varepsilon_{e}$ using Eq. (20),

$$
-N \kappa\left(v+\dot{\varepsilon}_{a}\right)+t-\frac{\partial \Psi^{p}}{\partial \lambda}-\Psi^{e} \frac{\partial N}{\partial \varepsilon_{a}}=\eta \dot{\varepsilon}_{a} .
$$


Next, make the following constitutive choice,

$$
\eta=\frac{1}{\beta}(t-\beta N \kappa+\alpha)
$$

where $\alpha, \beta>0$ are constants and introduce the maximum isometric stress

$$
t_{0}=\kappa v,
$$

which will be motivated later. Substitute Eqs. (26) and (25) into (24),

$$
t-N t_{0}-g=\frac{1}{\beta}(t+\alpha) \dot{\varepsilon}_{a},
$$

where $g=\partial \Psi^{p} / \partial \lambda+\Psi^{e}\left(\partial N / \partial \varepsilon_{a}\right)$. By a rearranging the terms, we arrive at

$$
\dot{\varepsilon}_{a}=\beta \frac{t-N t_{0}}{t+\alpha}-\beta \frac{g}{t+\alpha} .
$$

Quick-release experiments are generally performed in the maximum overlap region (McMahon 1984) which implies $N=1$ and $\partial N / \partial \varepsilon_{a}=0$ by Eq. (23). In addition, the passive part of the total force in Eq. (20) is given by $\partial \Psi^{p} / \partial \lambda$ and its contribution is generally small in this region for skeletal muscle (Böl 2010; Ehret et al. 2011; Gordon et al. 1966; Davis et al. 2003); an observation which holds here too. The passive part may, therefore, be neglected. Theses two observations imply $g=0$ and Eq. (28) simplifies to

$$
\dot{\varepsilon}_{a}=\beta \frac{t-t_{0}}{t+\alpha} .
$$

Equation (29) is very similar to the Hill equation in (1) apart from the sign of the nominator and the strain rate on the left-hand side instead of the shortening velocity. The sign of the nominator is simply related to extension being considered positive herein, contrary to Eq. (1). For the left-hand side, note that the model is given in terms of strain and stretch rather than length. The shortening velocity should, therefore, be replaced by the deformation rate $\dot{\lambda}$. From Eq. (3) it is seen that $\dot{\lambda}$ is the sum of two parts: a cross-bridge contraction rate $\dot{\varepsilon}_{e}$ and a filament sliding rate $\dot{\varepsilon}_{a}$. To be consistent with quick-release experiments, the shortening velocity is defined to be the slope of the stretch response curve just after the rapid shortening phase $\Delta \varepsilon_{e}$ following the quick release, see Fig. 2. The rapid shortening is associated with an elastic recoil in the cross-bridges (McMahon 1984) and by assuming the elastic length change to be instantaneous, $\dot{\varepsilon}_{e}=0$ immediately after the rapid shortening and the left-hand side becomes $\dot{\lambda}=\dot{\varepsilon}_{a}$ as a consequence. Equation (29) is, hence, equal to the Hill equation. Finally, to guarantee $\eta>0$ in the quick-release experiment, an upper bound for $\kappa$ can be established by substituting the minimum developed force $t=0$ and $N=1$ in Eq. (25). The result reads

$$
\kappa<\frac{\alpha}{\beta} .
$$



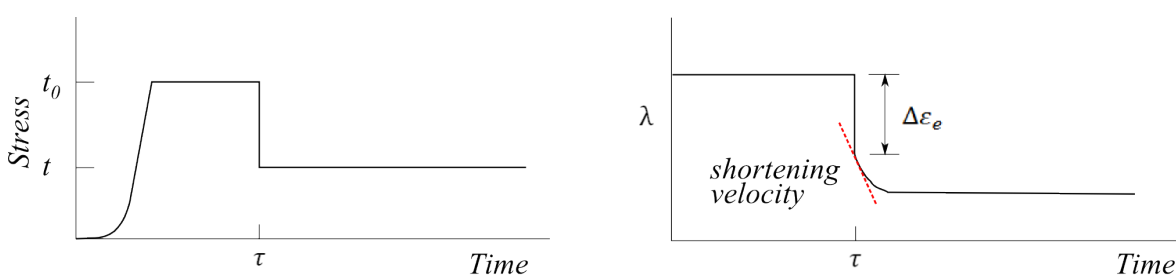

Fig. 2 A schematic representation of the quick-release experiment. The muscle is released from its isometric stress $t_{0}$ against a constant stress $t$ (left panel). The stretch response initially has a rapid shortening $\left(\Delta \varepsilon_{e}\right)$ followed by a gradual decline in the contraction velocity until a new steady state is reached.

\section{Parameter identification}

The constitutive model described in Sec. 2 includes a set of unknown parameters $\left(t_{0}, \lambda^{o p t}, \varepsilon_{a}^{o p t}, C_{1}, C_{2}, K_{e}, \kappa, v, \gamma, \alpha\right.$ and $\left.\beta\right)$ which must be determined for the specific muscle in question. The parameters are computed in a nonlinear identification process by tuning the model response to experimental data for the rabbit tibialis anterior muscle from Winters et al. (2009) and Jarvis and Salmons (1991). The data in Jarvis and Salmons (1991) is given in terms of force and velocity and must be adapted to the model. This is done by normalizing the force with the cross-sectional area $0.59 \mathrm{~cm}^{2}$ (Lieber and Blevins, 1989) and dividing the velocity by the initial muscle length $71.4 \mathrm{~mm}$ (Jarvis and Salmons, 1991). The parameter identification is done using the function fmincon in Matlab 7.13 (MathWorks, Natick, MA, USA) following the method described below.

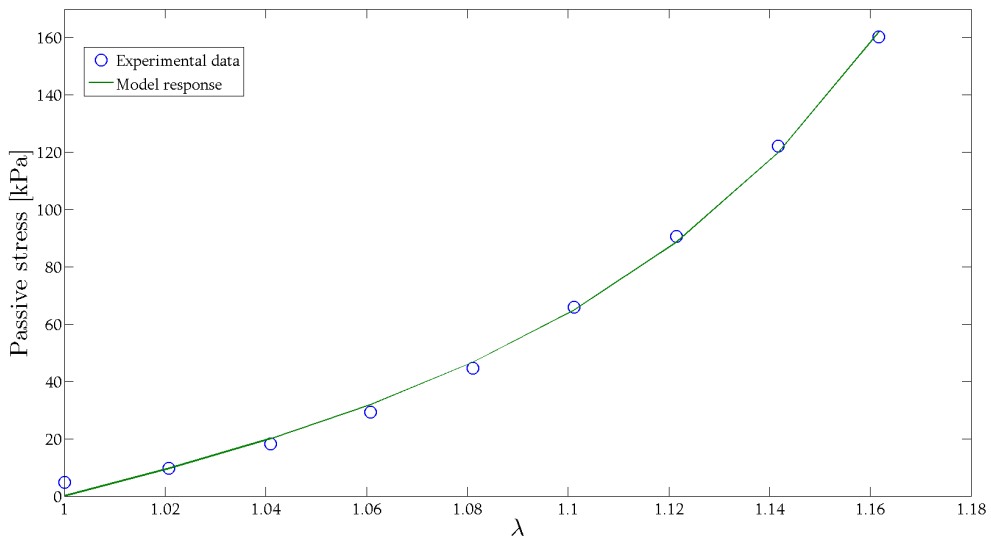

Fig. 3 The passive stress in the rabbit tibialis anterior muscle. The solid line is the model response and the circles are experimental data from Winters et al. (2009). 
First, substitute Eqs. (21) and (22) into (20) and compute the developed stress

$$
t=2 C_{1} \lambda\left(\lambda^{2}-1\right) \exp \left(C_{2}\left(\lambda^{2}-1\right)^{2}\right)+N K_{e} \varepsilon_{e}
$$

Second, the passive strain-energy function parameters $C_{1}$ and $C_{2}$ in Eq. (21) are identified by setting $K_{e}=0$ and fitting the passive stress part of Eq. (31) to the experimental passive stress data in Winters et al. (2009), see, Fig. 3.

Third, to obtain the active stress response, the experimentally measured passive stress is subtracted from the developed (tetanized) stress, see Fig. 4. The maximum point of this bell-shaped active stress curve is taken to be the maximal isometric stress $t_{0}$ and the corresponding stretch is $\lambda^{o p t}=1.012$ (Winters et al., 2009; Ehret et al., 2011), where a superscribed opt denotes the value at the maximum point.

Fourth, the parameters $\varepsilon_{a}^{o p t}, K_{e}, \kappa, v$ and $\gamma$ are obtained by subtracting the passive part from Eq. (31) and fitting $N K_{e} \varepsilon_{e}$ to the active stress data in Fig. 4 using Eqs. (2) and (23). The unknown variable $\varepsilon_{a}$ in Eq. (2) can be computed by substituting Eq. (19) ${ }^{1}$ into (18) and noting that $\dot{\varepsilon}_{a}=0$ since the data points in Fig. 4 obtained at steady state (Winters et al., 2009). This gives

$$
-N \kappa v-\Psi^{e} \frac{\partial N}{\partial \varepsilon_{a}}+N \frac{\partial \Psi^{e}}{\partial \varepsilon_{e}}=0
$$

from which $\varepsilon_{a}$ can be solved for each $\lambda$.

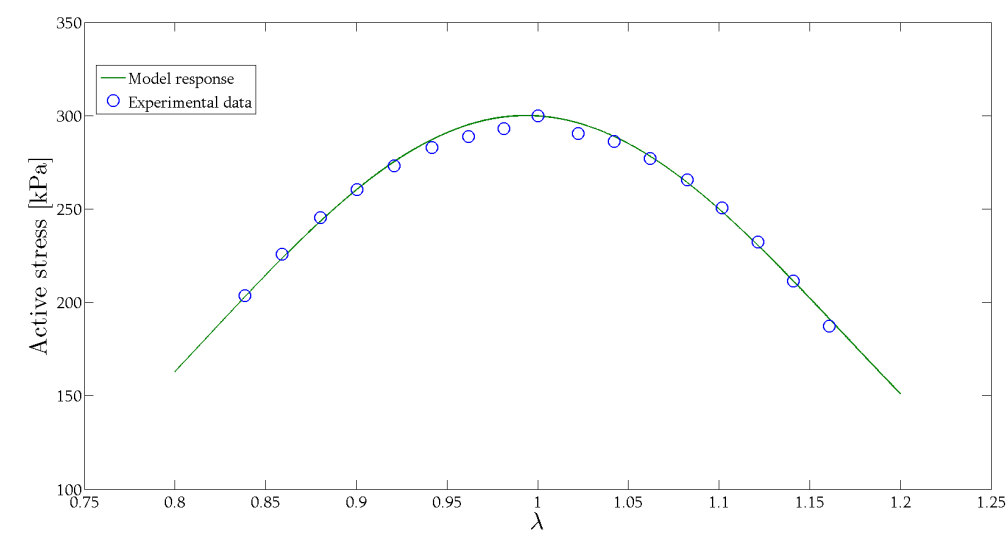

Fig. 4 The active stress data for rabbit tibialis anterior muscle. The solid line is the model response and the circles are experimental data from (Winters et al., 2009).

Finally, by fitting Eq. (29) to the experimentally observed stress-deformation rate curve in Jarvis and Salmons (1991), $\alpha$ and $\beta$ are identified, see, Fig. 5. The parameter identification result is summarized in Table 1.

Because the maximum point on the active stress curve is identified with $t_{0}$, the parameter $K_{e}$ becomes constrained in the parameter identification and 


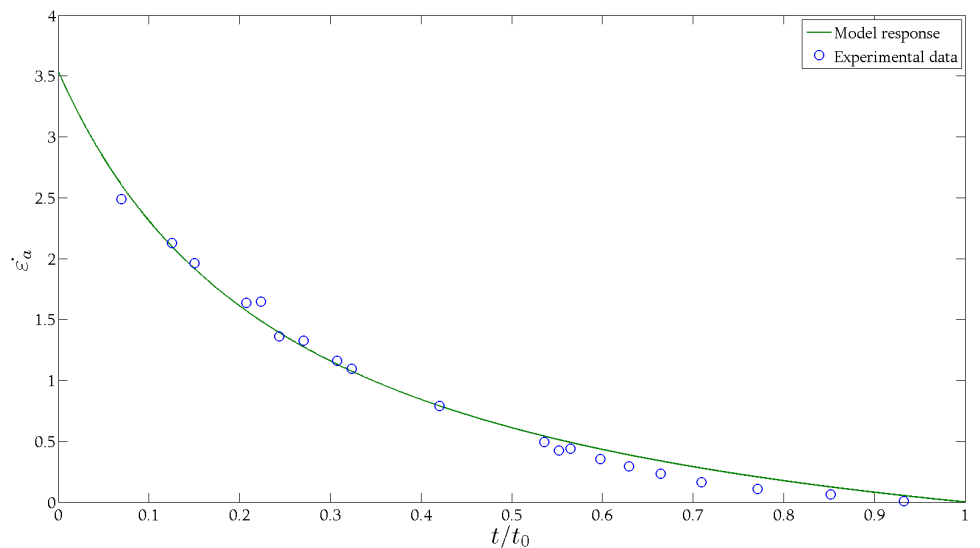

Fig. 5 The stress-strain-rate response for rabbit tibialis anterior muscle. The solid line is the model response and the circles are data from Jarvis and Salmons (1991).

Table 1 Parameters identified for the model based on the data in (Winters et al. 2009) and (Jarvis and Salmons 1991). The parameters $\kappa$ and $v$ are not shown since their product equal the maximum isometric stress by Eq. (34).

\begin{tabular}{ccccccccc}
\hline$t_{0}$ & $\lambda^{\text {opt }}$ & $\varepsilon_{a}^{\text {opt }}$ & $C_{1}$ & $C_{2}$ & $K_{e}$ & $\gamma$ & $\alpha$ & $\beta$ \\
{$[\mathrm{MPa}]$} & {$[-]$} & {$[-]$} & {$[\mathrm{MPa}]$} & {$[-]$} & {$[\mathrm{MPa}]$} & {$[-]$} & {$[\mathrm{Mpa}]$} & {$[1 / \mathrm{s}]$} \\
\hline 0.300 & 1.012 & -0.026 & 0.112 & 4.736 & 7.895 & 0.171 & 0.079 & 0.932 \\
\hline
\end{tabular}

the following equation must hold for the active stress,

$$
t_{0}=K_{e} \varepsilon_{e}^{o p t},
$$

where $\varepsilon_{e}^{o p t}=\lambda^{o p t}-1-\varepsilon_{a}^{o p t}$ by Eq. (2) and $N=1$. This equation can be used to eliminate $K_{e}$ from the parameter identification altogether. In addition, the steady-state assumption above implies a second constraint, namely

$$
t_{0}=\kappa v .
$$

This is realized by substituting $\partial N / \partial \varepsilon_{a}=0$ at $\varepsilon_{a}^{o p t}$ and Eq. (22) into (32), and comparing the result to Eq. (33). Note that Eq. (34) is the maximum isometric stress introduced in Sec. 2.4 and, since both $\kappa$ and $v$ are unknown, only their product can be determined uniquely in the parameter identification. As a consequence, Table 1 only gives the isometric stress.

\section{Discussion}

In this paper, we present a new model for skeletal muscle contraction. The model is derived by applying fundamental principles in mechanics and the active force does not rely on direct multiplication of the isometric stress by experimentally motivated functions; an approach used in many other studies, 
e.g., Martins et al. (1998), Johansson et al. (2000), and Liang et al. (2006). The active force is instead given by a set of nonlinear equations obtained from a virtual power balance and the dissipation inequality. Although several constitutive assumptions in the model are based on experimental results, there is a fundamental difference between this model and models where the isometric force is multiplied by arbitrary functions: the constitutive assumptions herein are chosen subject to the dissipation inequality (13). This implicitly guarantees a thermodynamic consistent behavior, which need not be the case when the isometric stress is multiplied by experimentally motivated functions.

It is difficult to assess if the identified parameters in Table 1 are reasonable since no similar model has been presented to the best of the authors' knowledge. The parameters $\alpha$ and $\beta$ in the Hill equation are an exception, however. They can be compared to previously reported values in the literature by using the normalized shortening velocity defined by (McMahon, 1984)

$$
\overline{\dot{\varepsilon}}_{a}=\frac{(\bar{t}-1)}{(1+\bar{t} / \xi)},
$$

where $\overline{\dot{\varepsilon}}_{a}=\dot{\varepsilon}_{a} / \dot{\varepsilon}_{a}^{\max }, \bar{t}=t / t_{0}$, and $\xi$ is a non-dimensional parameter given by $\xi=\alpha / t_{0}=\beta / \dot{\varepsilon}_{a}^{\max }$. The constant $\dot{\varepsilon}_{a}^{\max }=3.5 \mathrm{~s}^{-1}$ is the maximum shortening velocity obtained for $t / t_{0}=0$ in Fig. 5 . By substituting $t_{0}$ and $\alpha$ into Eq. (35), the dimensionless parameter is computed to be $\xi=0.26$ which is at the upper end of the normal interval $0.15<\xi<0.25$ for vertebra muscle (McMahon 1984). The same result holds true if $\xi$ is computed using $\dot{\varepsilon}_{a}^{\max }$ and $\beta$ instead.

Figures 3 to 5 show that the model is able to capture both passive and active properties of the rabbit tibialis anterior muscle. The poor fit of the Hill equation at small shortening velocities in Fig. 5 when compared to the same graph in Jarvis and Salmons (1991), is probably related to the data. The fitting of the Hill equation in Jarvis and Salmons (1991) was not constrained to go through the isometric force. As a consequence, $t_{0}$ in Eq. (29) should be replaced by the stress where the graph in Jarvis and Salmons (1991) intersects the force axis, which is about $270 \mathrm{kPa}$. For this value of $t_{0}$, the model shows a very good fit to data (not shown). Despite this we choose to present results for $t_{0}=300 \mathrm{kPa}$ since data is obtained from various sources. In an experiment tailored to the model, the measured isometric force will be the same in Figs. 4 and 5 .

The overlap function $N$ is chosen to be a normal distribution since it is a simple non-zero function with a continuous derivative. Despite its simplicity, the normal distribution captures the behavior of the active stress in Fig. 4 quite well and indicates that more elaborate functions for $N$, like the one used in Ehret et al. (2011), may not be needed for the tibialis anterior muscle in this model.

The strain-energy function $\psi^{e}$ is assumed to be quadratic in $\varepsilon_{e}$. In Huxley (1974) it was found that the stress response is close to linear. This motivates a quadratic strain energy if the serial spring represents the cross-bridges. This assumption requires the filaments to be rigid which is supported by X-ray diffraction studies, see McMahon (1984). If the serial spring is also taken to 
include other elastic structures in series with the cross-bridges, the linearity may be questioned. For example, the rigid filaments assumption has been contested by Huxley et al. (1994) who suggested a nonlinear stress response for the filaments. Nonetheless, we confine ourselves to a linear stress response as a first approximation to limit the complexity of the model.

The evolution law for $\varepsilon_{a}$ in Eq. (18) can be specialized to recover the Hill equation as shown in Sect. 2.4. This specialization restricts the model to shortening, however. Further, the shortening velocity is given by $\dot{\lambda}=\dot{\varepsilon}_{a}$ which requires $\dot{\varepsilon}_{e}=0$. From a mechanical point-of-view, this is a reasonable assumption in quick-release experiment since the serial spring can change its length instantaneously. This means that (most of) the rapid drop in length following the release is associated with the elastic cross-bridges. For slow contraction where the time constant is of the same order magnitude as the normalized clutch velocity $v$, the shortening velocity must be computed from the full problem using Eq. (3).

As pointed out in Sect. 3, only the product $\kappa v$ can be determined uniquely in the parameter identification. This is obviously a limitation, but it is not easy to think of ways to control $\kappa$ and $v$ independently in the experiments behind Fig. 4. Nonetheless, upper and lower bounds for $\kappa$ and $v$, respectively, can be determined. The first bound $\kappa<\alpha / \beta$ is already given by Eq. (30). The second bound is readily computed by substituting the definitions $\alpha=\xi t_{0}$ and $\beta=\xi \dot{\varepsilon}_{a}^{\max }$ (see Eq. (35)) into Eq. (30) which gives

$$
\frac{v}{\dot{\varepsilon}_{a}^{\max }}>1 .
$$

The implication of Eq. (36) is that the friction-clutch's normalized velocity $v$ must exceed the maximum shortening velocity to guarantee dissipation for active contraction, i.e., $\eta>0$. These bounds may be used in simulations where explicit values for $\kappa$ and $v$ are needed, but it comes at a cost since $\eta$ in Eq. (24) becomes zero and the contraction is non-dissipative. If one is willing to accept a pragmatic position, this may be of minor importance in most (practical) applications where shortening takes place in the presence of a load, i.e., $t \neq 0$, and the maximum shortening velocity is never reached.

The presented model is limited to contraction of fully tetanized muscle. If it is desirable to include partial activation in the model, it is possible to use the method described in Stålhand et al. (2008). Briefly, the model is extended by introducing variables for the internal calcium ion concentration and the fraction of myosin heads attached to actin (cross-bridges). Appropriate modification of the external and internal virtual powers and the strain energies gives an additional evolution equation describing the fraction of attached myosin as a function of the calcium ion concentration. We chose to exclude partial activation from the model since it only adds to the complexity and most quick-release experiments are performed with fully tetanized muscles anyway.

In conclusion, a new model for skeletal muscle contraction is presented in this paper. The model is based on an additive decomposition of the deformation and is derived subject to fundamental mechanical principles. The model 
shows good agreement with experimental data and we believe it can contribute to our understanding on how to model the active properties of skeletal muscles.

Acknowledgements This work was financed by the Swedish Research Council and their support is gratefully acknowledged. The authors also wish to thank Prof. A. Klarbring and Dr. J. Holmberg at Linöping University for their valuable comments.

\section{References}

Ambrosi D, Pezzuto S (2012) Active stress vs. active strain in mechanobiology: constitutive issues. J Elasticity 107(2):199-212

Buchanan TS, Lloyd DG, Manal K, Beiser TF (2004) Neuromusculoskeletal modeling: estimation of muscle forces and joint moments and movements from measurements of neural command. J Appl Biomech 20:367-395

Böl M (2010) Micromechanical modeling of skeletal muscles: from the single fiber to the whole muscle. Arch Appl Mech 80:557-567

Davis J, Kaufman KR, Lieber RL (2003) Correlation between active and passive isometric force and intramuscular pressure in the isolated rabbit tibialis anterior muscle. J Biomech 36:505-512

Edman KAP, Elzinga G, Noble MIM (1982) Residual force enhancement after stretch of contracting frog single muscle fibers. J Gen Physiol 80:796-784

Ehret AE, Itskov M (2007) A polyconvex hyperelastic model for fiberreinforced materials in application to soft tissues. J Mater Sci 42:8853-8863

Ehret AE, Böl M and Itskov M (2011) A continuum constitutive model for the behaviour of skeletal muscle. J Mechanics and Physics of Solids 59:625-636

Ettema GJC, Meijer K (2000) Muscle contraction history: modified Hill versus an exponential decay model. Cybernetics 83:491-500

Fung YC (1993) Biomechanics. Mechanical properties of living tissues. 2 ed, Springer, New York

Fung YC, Fronek K, Patitucci P (1979) Pseudoelasticity of arteries and the choice of its mathematical expression. Am J Physiol 237:H620-H631

Germain P (1973) The Method of Virtual Power in Continuum Mechanics. Part 2: Microstructure, SIAM J Appl Math, Vol25(3):556-575

Gordon AM, Huxley AF, Julian FJ (1966) The variation in isometric tension with sarcomere length in vertebrate muscle fibers. J Physiol 184:170-192

Hill AV (1938) The heat of shortening and the dynamic constants of muscle. Proc Roy Soc B 126:136-195

Huxley AF (1974) Muscular contraction (review lecture). J Physiol (Lond) 243:1-43

Huxley HE, Stewart A, Sosa H, Irving T (1994) X-ray diffraction measurements of the extensibility of actin and myosin filaments in contracting muscle. Biophys J 67:2411-2421

Huxley AF, Tideswell S (1996) Filament compliance and tension transients in muscle. J Muscle and Cell Motility 17:507-511 
Jarvis JC, Salmons S (1991) An electrohydraulic apparatus for the measurement of static and dynamic properties of rabbit muscles. J Appl Physiol 70:938-941

Johansson T, Meier P, Blickhan R (2000) A finite-element model for the mechanical analysis of skeletal muscles. J Theoretical Biology 206:131-149

Liang Y, McMeeking RM, Evans AG (2006) A finite element simulation scheme for biological muscular hydrostats. J Theoretical Biology 242:142-150

Lieber RL, Blevins FT (1989) Skeletal muscle architecture of the rabbit hindlimb: functional implications of muscle design. J Morphol 199:93-101

Martins JAC, Pires EB, Salvado R, Dinis PB (1998) A numerical model of passive and active behavior of skeletal muscles. Computer Methods in Applied Mechanics and Engineering 151:419-433

McMahon TA (1984) Muscles, Reflexes, and Locomotion. Princeton University Press

Meijer K, Grootenboer HJ, Koopman HFJM, Linden BJJJ, Huijing PA (1998) A Hill type model of rat medial gastrocnemius muscle that accounts for shortening history effects-performance of excitation dynamics optimized for a twitch in predicting tetanic muscle forces. J Biomech 31:555-563

Murtada SC, Arner A, Holzapfel GA (2011) Experiments and mechanochemical modeling of smooth muscle contraction: Significance of filament overlap. J Theoretical Biology 297:176-186

Murtada SI, Kroon M, Holzapfel GA (2010) A calcium-driven mechanochemical model for prediction of frog generation in smooth muscle. Biomech Model Mechanobiol 9:749-762

Stålhand J, Klarbring A, Holzapfel GA (2008) Smooth muscle contraction: Mechanochemical formulation for homogeneous finite strains. Biophysics and Molecular Biology 96:465-481

Stålhand J, Klarbring A, Holzapfel GA (2011) A mechanochemical 3D continuum model for smooth muscle contraction under finite strains. J Theoretical Biology 268:120-130

Winters TM, Sepulveda GS, Cotter PS, Kaufman KR, Lieber RL, Ward SR (2009) Correlation between isometric force and intramuscular pressure in rabbit tibialis anterior muscle with an intact anterior compartment. Muscle Nerve 40:79-85

Zajac FE (1989) Muscle and Tendon: properties, models, scaling, and application to biomechanics and motor control. Critical Reviews in Biomechanical Engineering 17(4):359-411 